\title{
Low-Energy Electron Escape from Liquid Interfaces: Charge and Quantum Effects
}

\section{Journal Article}

\section{Author(s):}

Ban, Loren (iD; Gartmann, Thomas E.; Yoder, Bruce L.; Signorell, Ruth (D)

Publication date:

2020-01-10

Permanent link:

https://doi.org/10.3929/ethz-b-000389640

Rights / license:

In Copyright - Non-Commercial Use Permitted

Originally published in:

Physical Review Letters 124(1), https://doi.org/10.1103/physrevlett.124.013402

\section{Funding acknowledgement:}

786636 - Droplet Photoelectron Imaging (EC)

172472 - Phase Transitions of Ultrafine Aerosol Particles: Condensation, Freezing, and Metal Formation in Confined Systems (SNF) 


\title{
Low-Energy Electron Escape from Liquid Interfaces: Charge and Quantum Effects
}

\author{
Loren Ban๑, Thomas E. Gartmann, Bruce L. Yoder, and R. Signorellø* \\ Department of Chemistry and Applied Biosciences, Laboratory of Physical Chemistry, \\ ETH Zürich, Vladimir-Prelog Weg 2, CH-8093 Zürich, Switzerland
}

\section{(Received 23 August 2019; published 10 January 2020)}

\begin{abstract}
The high surface sensitivity and controlled surface charge state of submicron sized droplets is exploited to study low-energy electron transport through liquid interfaces using photoelectron imaging. Already a few charges on a droplet are found to modify the photoelectron images significantly. For narrow escape barriers, the comparison with an electron scattering model reveals pronounced quantum effects in the form of above-barrier reflections at electron kinetic energies below about $1 \mathrm{eV}$. The observed susceptibility to the characteristics of the electron escape barrier might provide access to these properties for liquid interfaces, which are generally difficult to investigate.
\end{abstract}

DOI: 10.1103/PhysRevLett.124.013402

The interaction of low-energy electrons (LEE, $<50 \mathrm{eV}$ ) with condensed molecular matter is relevant to many fields, ranging from radiation damage of biological systems, to atmospheric chemistry and astrochemistry, to the engineering of electronic devices [1-6]. A phenomenon that has received particularly broad attention in this context is the formation of the solvated electron and the role it might play in radiation damage [7-16]. Experimental studies mainly concentrated on low-energy photoelectron transmission (LEPET) or low-energy electron transmission (LEET) spectroscopy of thin films [6], while liquid microjets, aerosol particles, and molecular clusters were later suggested as alternative samples for the investigation of LEE transport in dielectrics [9,17-30].

Interfacial electron transfer and the scattering processes it involves play a vital role in LEE transport [5,31-39], but they are particularly difficult to investigate experimentally in the case of (volatile) molecular liquids. Major obstacles arise from radiation-induced charging and the incompatibility of high-vacuum conditions with thin-film and bulk samples of high vapor pressure. As a result, there is still no consensus even on the liquid-vacuum interface potential of water [40-45], with reported values of the escape barrier typically varying between 0.1 and $1.2 \mathrm{eV}$. The situation is further complicated by the sensitivity of LEE escape from interfaces to the presence of even only a few charges. Corresponding studies are rather scarce $[46,47]$, presumably because it is difficult to control the exact charge state of thin-film or bulk samples. Small particles have been used to study electron impact or photoelectron charging mechanisms $[17,18,48-50]$, but to the best of our knowledge the LEE escape from the interface has not been investigated in detail.

Here, we present the results of a combined experimental and theoretical study of LEE transfer across liquid-vacuum interfaces. We use angle-resolved photoelectron spectroscopy of submicron-sized droplets to access information on the photoelectron kinetic energy (eKE) and the photoelectron angular distribution (PAD). Droplets offer important advantages over thin-film or bulk samples for such studies: (i) vacuum compatibility even for volatile molecular liquids, (ii) no radiation-induced sample charging because of constant sample refreshment, (iii) high surface sensitivity, and (iv) control over and independent determination of the charge state. This allows us to exploit the high sensitivity of LEE below a few $\mathrm{eV}$ to the properties of the interface potential and charge state. The comparison with an electron scattering model reveals that the photoelectron distribution is not only affected by the electrostatic interaction with the droplet charge. Depending on the characteristics of the interface potential quantum effects can play a significant role in LEE transfer through the liquid-vacuum interface.

Experiment.-Liquid dioctyl phthalate (DEHP) droplets were produced by atomization (TSI model 3076) of neat liquid DEHP, size selected in a differential mobility analyzer (TSI model 3081) and charged in a home-built corona-wire unipolar charger. Droplets with an average radius $\left\langle R_{D}\right\rangle$ of $\sim 210 \mathrm{~nm}$ were investigated in five different average charge states $\langle q\rangle=+16,+8,0,-7,-15$ (Figs. S3-S6 of the Supplemental Material [51]; $q$ is the number of elementary charges). The droplets were then transferred to vacuum by an aerodynamic lens (ADL) [52,53] and resonantly two-photon ionized by a $266 \mathrm{~nm}$ nanosecond laser. Photoelectron kinetic energies (eKEs) and angular distributions (PADs) were recorded with a velocity map imaging (VMI) spectrometer [54]. The resulting raw images (see below) provide a two-dimensional projection of the three-dimensional photoelectron velocity distribution. PADs were analyzed directly in terms of the raw images, while eKE spectra were retrieved from the three-dimensional velocity distribution reconstructed with MEVIR [55] (see Ref. [51]). 
(a)

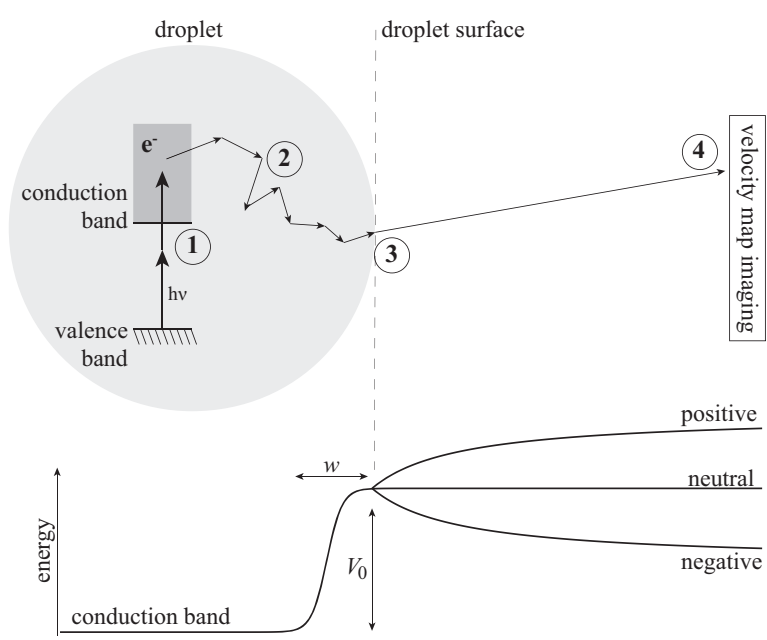

FIG. 1. (a) Photoionization (1), electron transport scattering (2), escape at the droplet-vacuum interface (3), and VMI detection (4). (b) Charge-dependent potential energy (excluding centrifugal potential [51]).

Electron scattering model.-The model for the photoionization [step 1 in Fig. 1(a)], electron transport scattering (step 2), electron escape at the droplet-vacuum interface (step 3), and detection by VMI (step 4) follows our previous work $[9,19,21,30]$ with extensions for the treatment of surface charges and electron escape at the dropletvacuum interface. A laser excites valence electrons into the conduction band (step 1). The genuine binding energy spectrum of DEHP required to describe this step was determined from a fit to the experimental spectrum of the neutral particles $[\langle q\rangle=0$ in Fig. 2(b)] excluding eKEs $\leq 0.1 \mathrm{eV}$ [51]. The obtained genuine spectrum (Fig. S7 in the Supplemental Material [51]) is in reasonable agreement with the calculated gas-phase spectrum of dimethyl phthalate with a gas-to-liquid shift of $\sim 2 \mathrm{eV}$, similar to benzene [56-58]. Electron transport scattering (step 2) was modeled with a probabilistic electron scattering model $[9,19,30]$, which amounts to a Monte Carlo solution of the transport equation. The requisite differential scattering cross sections (Fig. S8 [51]) were derived from data for benzene [59,60] as described in Ref. [51]. We assume charges to be uniformly distributed on the droplet's surface adding a constant potential inside the droplet [17] and a Coulomb potential outside to the neutral droplet's steplike barrier at the interface with height $V_{0}$ and width $w$ [Fig. 1(b)]. The effective potential experienced by the electron takes the following form:

$$
\begin{aligned}
V(r)= & \frac{V_{0}}{2}\left\{1+\tanh \left[\frac{2 a}{w}\left(r-R_{D}+\frac{w}{2}\right)\right]\right\} \\
& +V_{\text {Coulomb }}+V_{\text {centrifugal }},
\end{aligned}
$$

where $R_{D}$ is the droplet's radius, $r$ the radial distance from its center, and $a=\tanh ^{-1}(0.999)$. The first term describes the
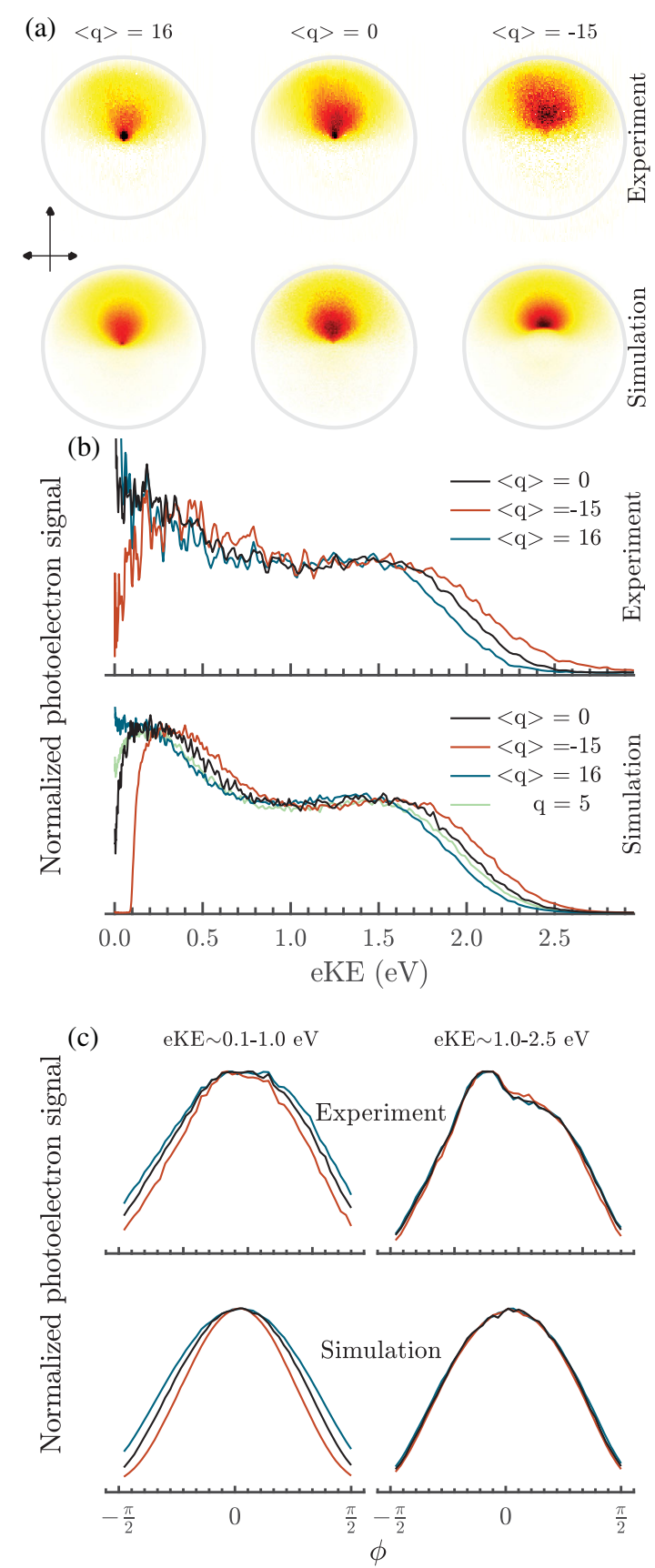

FIG. 2. (a) Experimental (top) and simulated (bottom) velocity map images of DEHP droplets. Red is high, yellow is low, and white is no electron intensity. The arrows indicate the laser propagation and polarization direction. The simulations are for $V_{0}=1 \mathrm{eV}$ and $w=0.1 \mathrm{~nm}$ and a genuine photoelectron spectrum obtained by a fit to the neutral experimental eKE spectrum. (b) eKE spectra retrieved for the neutral (black), positively (blue), and negatively charged droplets (red). Additional simulation for a charge of $q=5$ (green). The spectra are normalized to their respective values at $1.5 \mathrm{eV}$. (c) Angular distribution of the integrated low (left) and high (right) eKE signal for neutral (black), positively (blue), and negatively charged droplets (red) (see text and Ref. [51]). $\phi$ is the angle with respect to the direction of light propagation. Distributions are normalized to their respective values at $\phi=0$. 
bare potential barrier with height $V_{0}$ and width $w . V_{0}$ specifies the position of the vacuum relative to the bottom of the conduction band for the neutral particle. $w$ defines the width over which the barrier reaches $99.9 \%$ of $V_{0}$. $V_{\text {Coulomb }}$ and $V_{\text {centrifugal }}$ are the Coulomb and centrifugal potentials, respectively (see the Supplemental Material [51]).

To account for quantum effects we calculate the transmission probability $T$ at the interface from the numerical solution of the radial Schrödinger equation for the given potential. As a consequence of centrifugal potential contributions, $T$ depends on the impact angle $\theta$ of the electron relative to the surface normal [Fig. 3(c) and Ref. [51]).

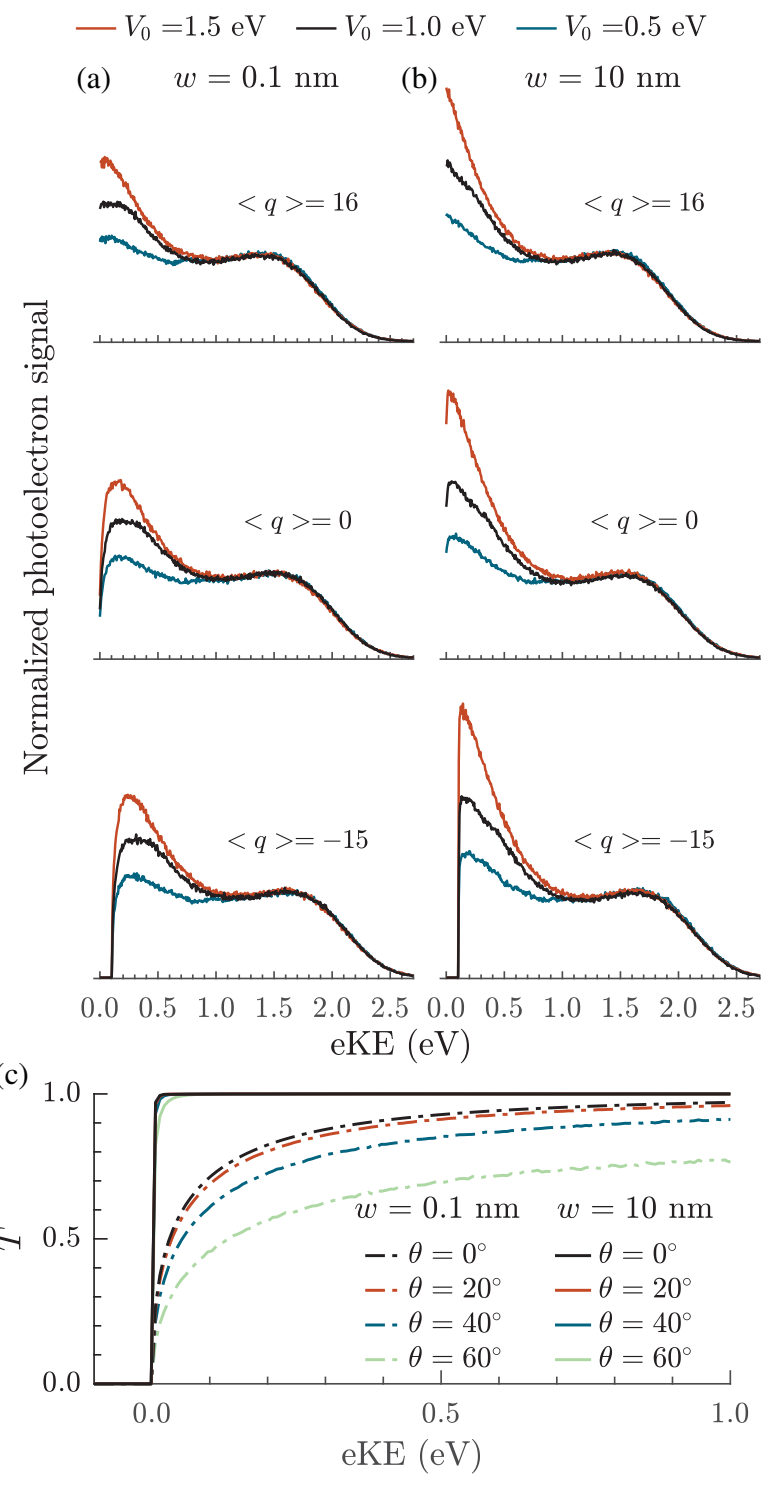

FIG. 3. Simulated eKE spectra for (a) $w=0.1$ and (b) $w=10 \mathrm{~nm}$. Blue line: $V_{0}=0.5$, black line: $V_{0}=1.0$, red line: $V_{0}=1.5 \mathrm{eV}$. The same genuine properties are used for all simulations. (c) Electron transmission coefficient $T$ for $V_{0}=$ $1.0 \mathrm{eV}$ and $\langle q\rangle=0$ as a function of the eKE for $w=0.1$ (dashdotted lines) and $w=10 \mathrm{~nm}$ (solid lines) for different incidence angles $\theta$ (colors).
Following transmission, electrons are propagated classically before their final velocity is projected onto the detector plane to produce the image (VMI). Images simulated for different sizes and charge states are averaged according to the experimentally determined size and charge distributions [51].

Results and discussion.-Figure 2(a) shows experimental photoelectron VMIs (top row) for droplets with average charge states $\langle q\rangle=+16$ (left), 0 (center) and -15 (right), respectively. As a result of nanofocusing, the images show strong asymmetry along the laser propagation direction with higher electron intensity opposite the illuminated side of the droplet [19]. Variation of the charge state from positive to negative decreases the near zero eKE electron signal (near the image center) and increases that of higher eKE electrons (towards the image border). This is accompanied by a change in the angular distribution of the electron signal. The trends become clearer in the eKE spectra [Fig. 2(b), top row] and PADs [Fig. 2(c), top row] retrieved from the images. Generally, good agreement is found between experiments and scattering simulations [Figs. 2(a), 2(b), 2(c), bottom row]. Based on their charge dependence, the spectra can be divided into a high $(>1 \mathrm{eV})$ and a low energy $(<1 \mathrm{eV})$ region:

High eKE region $(>1 \mathrm{eV})$ : The main feature in the $\mathrm{eKE}$ spectra [Fig. 2(b)] is the charge-dependent energy shift of the high eKE onset (Table I and Ref. [51]), which reflects the corresponding shift of the vacuum level (Fig. 1). Relative to the neutral case, spectra of positively charged droplets are shifted towards lower eKE by up to $100 \mathrm{meV}$, while we observe shifts to higher eKE of up to $250 \mathrm{meV}$ for negative charges. Most of the observed shifts of the high eKE edge agree with the corresponding calculated average shift of the vacuum level which is proportional to $\langle q\rangle$ (Fig. S9 [51]). In this region the electron's kinetic energy significantly exceeds both the barrier height and even more so the Coulomb shift of the vacuum level. The higher the eKE value lies above the top of the barrier, the more classical the transmission becomes. As a consequence the shape of the high eKE edge is neither pronouncedly influenced by quantum effects (see below) nor by the shape of the barrier $\left(V_{0}\right.$ and $\left.w\right)$. The latter is illustrated by

TABLE I. Charge-dependent high kinetic energy onset (eKE onset) determined at the $1 / e^{2}$ signal level [51] and energy shift $\Delta \mathrm{eKE}$ relative to the neutral case.

\begin{tabular}{rccccc}
\hline \hline & \multicolumn{2}{c}{$\mathrm{eKE}$ onset $(\mathrm{eV})$} & & \multicolumn{2}{c}{$\Delta \mathrm{eKE}(\mathrm{eV})$} \\
\cline { 2 - 3 } \cline { 5 - 6 }$\langle q\rangle$ & Experiment & Simulation & & Experiment & Simulation \\
\hline 16 & 2.21 & 2.21 & & -0.11 & -0.10 \\
8 & 2.26 & 2.25 & & -0.06 & -0.06 \\
0 & 2.32 & 2.31 & & 0 & 0 \\
-7 & 2.40 & 2.37 & & 0.08 & 0.06 \\
-15 & 2.57 & 2.43 & & 0.25 & 0.12 \\
\hline \hline
\end{tabular}


the simulations for different values of $V_{0}$ and $w$ which leave the high eKE region unaffected (Fig. 3). We note that changing $V_{0}$ or $w$ cannot shift the photoelectron spectrum on the eKE axis (as the surface charge does) but only affect relative intensities in the spectrum.

Good agreement between experimental and simulated PADs is reflected in the velocity map images [Fig. 2(a)]. This is illustrated in Fig. 2(c) for the charge-dependent angular distribution of the integrated low (left column) and high (right column) eKE signal. The traces were obtained by integrating the images for each given direction over electron velocities in the detector plane corresponding to eKEs in the range $0.1-1.0 \mathrm{eV}$ and $1.0-2.5 \mathrm{eV}$, respectively. The direction is specified in terms of the angle $\phi$ with respect to the direction of light propagation. Contrary to the eKE spectra, no significant charge dependence is observed in the high eKE range between 1.0-2.5 eV [Fig. 2(c), right column and Fig. S10 in Ref. [51]). (The asymmetry with respect to $\phi=0$ in the experimental images for eKEs between $1.0-2.5 \mathrm{eV}$ is due to inhomogeneities of the electron imaging detector.)

Low eKE region $(<1 \mathrm{eV})$ : Negative charges lead to significantly lower electron signals at low eKE compared with neutral and positive charges [Fig. 2(b)]. Even though this general trend is captured by the simulation, there are deviations between simulation and experiment, in particular for the neutral droplets. They can be partly attributed to the larger experimental uncertainties in this low eKE range and to difficulties in generating neutral droplets. Compared with higher eKE electrons, it is more challenging to quantitatively record very low eKE electrons, which are much more sensitive to small perturbations (e.g., external fields, imperfections in the VMI optics) affecting the measured eKE values. Furthermore, the lower angular resolution in the center of the electron detector strongly reduces the signal to noise level for near zero eKE electrons. Additional limitations arise for the neutral case, where the simulation predicts a smaller near zero eKE signal than found in the experiment. It is challenging to generate completely uncharged droplets, and difficult to quantify the exact charge state of droplets with very few charges on them. All this is exacerbated by the high sensitivity of the photoelectron spectrum in the low eKE region to the presence of even a small number of charges. This is illustrated by the simulation for $q=5$ in Fig. 2(b) (green line). Already a small amount of positive charges considerably increases the signal near zero eKE compared to uncharged particles.

The observed charge-dependence of the spectra in the low eKE region results from the electrostatic interactions of the electrons with the charged droplet surface following the escape. The main effect is the overall shift of the photoelectron spectrum already discussed above for the high kinetic energy edge. In contrast to high eKE electrons, the relatively large de Broglie wavelength $(\sim 1.7 \mathrm{~nm}$ at $0.5 \mathrm{eV})$ of low eKE electrons makes them very sensitive to quantum effects and the exact shape $\left(V_{0}\right.$ and $\left.w\right)$ of the interface potential. Simulated eKE spectra for different barrier heights $\left(V_{0}=0.5,1.0\right.$, and $\left.1.5 \mathrm{eV}\right)$ are shown in Figs. 3(a) and 3(b) for two limiting cases of the barrier widths $w=0.1$ and $10 \mathrm{~nm}$, respectively. The latter is certainly broader than would appear physically plausible. Here it only serves to illustrate a limiting (classical) behavior. A higher barrier is equivalent to a lower bottom of the conduction band and hence higher eKE of the electron inside the droplet. In this way, the relative abundance of low eKE electrons increases with $V_{0}$ as a consequence of the energy-dependence of the electron scattering cross sections (Fig. S7 [51]). A narrow, steplike barrier $[w=0.1 \mathrm{~nm}$, Fig. 3(a)] represents a case where quantum effects on the electron escape play a prominent role. These effects are suppressed in the case of a broad, smooth barrier $[w=10 \mathrm{~nm}$, Fig. 3(b)]. Irrespective of the barrier height $V_{0}$, the relative abundance of low eKE electrons is lower for the narrow barrier $(w=0.1 \mathrm{~nm})$, where quantum (above-barrier) reflections reduce the electron transmission through the interface. For a fixed barrier height $V_{0}=1 \mathrm{eV}$ and charge state $\langle q\rangle=0$, Fig. 3(c) shows the transmission coefficient $T$ as a function of eKE for $w=0.1$ (dashed lines) and $w=10 \mathrm{~nm}$ (full lines) for different incidence angles $\theta$ (angle relative to the surface normal). Quantum reflections in the case of the narrow barrier strongly reduce $T$ for eKE $<1 \mathrm{eV}$, while the broad barrier produces an almost classical transmission behavior. This holds regardless of the droplet charge, though it is most evident for the negatively charged droplets, where the sharp signal cutoff for $w=10 \mathrm{~nm}$ transforms into a smoother decrease for $w=0.1 \mathrm{~nm}$ [Figs. 3(a) and 3(b)]. With increasing barrier height $V_{0}$, the difference between the eKE spectra for $w=0.1$ and $10 \mathrm{~nm}$ becomes more pronounced as a result of the stronger reduction of $T$ for higher barriers (Fig. S11 and S12 [51]). Significant contributions to the electron transmission arising from tunneling (as opposed to quantum reflections) are not found in the simulations, as expected for the relatively broad potential cusp at the barrier (Fig. 1).

In contrast to the high eKE region [ $>1 \mathrm{eV}$, Fig. 2(c), right], the angular distributions of low eKE signal $[<1 \mathrm{eV}$, Fig. 2(c), left] is sensitive to the droplet charge. Fig. S11 [51] reveals that the highest sensitivity arises for the lowest eKE values $(<0.5 \mathrm{eV})$, i.e., the region where quantum transmission effects are pronounced [Fig. 3(c)]. The general trend is a reduction of the degree of forward scattering from negative to neutral to positive charge. Most of the effect has a classical origin: electrons are accelerated (or decelerated) by the Coulomb potential. If they exit the droplet at a nonvanishing angle to the surface normal (impact angle $\theta$, finite angular momentum) negative charges (acceleration) will reduce and positive charges (deceleration) increase that angle compared with the neutral case. As most electrons are 
ejected in the direction of light propagation, negative (positive) charges will on balance reduce (increase) the relative abundance of electrons moving orthogonal to the direction of light propagation, which is what we observe. Although largely classical, this effect is enhanced by above-barrier reflection, which preferentially reduces the transmission of electrons with large impact angles $\theta$. This quantum effect becomes more pronounced in going from positive to negative charging of the droplets $(\theta=0$ in Fig. S11 vs $\theta=60^{\circ}$ in Fig. S12 [51]).

Conclusion.-Angle-resolved photoelectron spectroscopy of neutral, positively, and negatively charged droplets at electron kinetic energies below a few $\mathrm{eV}$ provides information on the electron escape from liquid interfaces. From the position of the high kinetic energy onset of the electron signal, we can determine charge-dependent binding (ionization) energies-which have been elusive to experimental probes for liquids. At very low kinetic energies $(<1 \mathrm{eV})$ the photoelectron spectra are highly sensitive to the exact charge state as well as the height and width of the electron escape barrier. The quantum effect of above-barrier reflections at narrow barriers strongly reduces the abundance of very low kinetic energy electrons compared with broader barriers. The comparison with an electron scattering model shows that tunneling-in contrast to reflection-does not significantly contribute to the observed photoelectron spectra. So far, consistent experimental values of barrier heights and widths are not available for most liquids, including water. The droplet approach might provide new experimental access to escape barrier properties even for such liquid interfaces. Furthermore, droplets offer a way to systematically investigate the influence of the charge state on the photoelectron spectra of liquids - an issue that is being discussed with regard to liquid-microjet photoelectron spectroscopy and more generally in the context of radiation-induced sample charging in condensed phase photoelectron spectroscopy.

We are very grateful to Dr. Martin Fierz for lending us a unipolar aerosol charger and for his advice concerning the aerosol charging experiments, and to Dr. David Luckhaus for his advice regarding the simulations. We thank David Stapfer, Markus Steger, and Daniel Zindel for technical support. This project has received funding from the European Unions Horizon 2020 research and innovation program from the European Research Council under the Grant Agreement No. 786636, and from the Swiss National Science Foundation (SNSF) through SNSF Project No. 200020_172472.

*rsignorell@ethz.ch

[1] E. Alizadeh, T. M. Orlando, and L. Sanche, Annu. Rev. Phys. Chem. 66, 379 (2015).

[2] M. Michaud, A. Wen, and L. Sanche, Radiat. Res. 159, 3 (2003).
[3] Q.-B. Lu and L. Sanche, Phys. Rev. Lett. 87, 078501 (2001).

[4] M. C. Boyer, N. Rivas, A. A. Tran, C. A. Verish, and C. R. Arumainayagam, Surf. Sci. 652, 26 (2016).

[5] C. Ferradini and J. Jay-Gerin, Excess Electrons in Dielectric Media (CRC Press, Boca Raton, 1991).

[6] R. Naaman and L. Sanche, Chem. Rev. 107, 1553 (2007).

[7] R. M. Young and D. M. Neumark, Chem. Rev. 112, 5553 (2012).

[8] M. H. Elkins, H. L. Williams, A. T. Shreve, and D. M. Neumark, Science 342, 1496 (2013).

[9] D. Luckhaus, Y.-I. Yamamoto, T. Suzuki, and R. Signorell, Sci. Adv. 3, e1603224 (2017).

[10] S. Karashima, Y.-I. Yamamoto, and T. Suzuki, J. Phys. Chem. Lett. 10, 4499 (2019).

[11] L. Ma, K. Majer, F. Chirot, and B. von Issendorff, J. Chem. Phys. 131, 144303 (2009).

[12] A. Lietard and J. R. R. Verlet, J. Phys. Chem. Lett. 10, 1180 (2019).

[13] T. E. Gartmann, L. Ban, B. L. Yoder, S. Hartweg, E. Chasovskikh, and R. Signorell, J. Phys. Chem. Lett. 10, 4777 (2019).

[14] L. Turi and P. J. Rossky, Chem. Rev. 112, 5641 (2012).

[15] J. M. Herbert and M. P. Coons, Annu. Rev. Phys. Chem. 68, 447 (2017).

[16] C.-C. Zho, V. Vlček, D. Neuhauser, and B. J. Schwartz, J. Phys. Chem. Lett. 9, 5173 (2018).

[17] P. J. Ziemann, P. Liu, D. B. Kittelson, and P. H. McMurry, J. Phys. Chem. 99, 5126 (1995).

[18] P. J. Ziemann, D. B. Kittelson, and P. H. McMurry, J. Aerosol Sci. 27, 587 (1996).

[19] R. Signorell, M. Goldmann, B. L. Yoder, A. Bodi, E. Chasovskikh, L. Lang, and D. Luckhaus, Chem. Phys. Lett. 658, 1 (2016).

[20] K. R. Wilson, Z. Shengli, S. Jinian, E. Rühl, S. R. Leone, G. C. Schatz, and M. Ahmed, Nano Lett. 7, 2014 (2007).

[21] M. Goldmann, J. Miguel-Sánchez, A. H. C. West, B. L. Yoder, and R. Signorell, J. Chem. Phys. 142, 224304 (2015).

[22] S. Amanatidis, B. L. Yoder, and R. Signorell, J. Chem. Phys. 146, 224204 (2017).

[23] M. I. Jacobs, O. Kostko, M. Ahmed, and K. R. Wilson, Phys. Chem. Chem. Phys. 19, 13372 (2017).

[24] L. Seiffert et al., Nat. Phys. 13, 766 (2017).

[25] S. Thürmer, R. Seidel, M. Faubel, W. Eberhardt, J. C. Hemminger, S. E. Bradforth, and B. Winter, Phys. Rev. Lett. 111, 173005 (2013).

[26] Y.-I. Suzuki, K. Nishizawa, N. Kurahashi, and T. Suzuki, Phys. Rev. E 90, 010302(R) (2014).

[27] J. Nishitani, C. W. West, and T. Suzuki, Struct. Dyn. 4, 044014 (2017).

[28] C. Zhang, T. Andersson, M. Förstel, M. Mucke, T. Arion, M. Tchaplyguine, O. Björneholm, and U. Hergenhahn, J. Chem. Phys. 138, 234306 (2013).

[29] T. E. Gartmann, S. Hartweg, L. Ban, E. Chasovskikh, B. L. Yoder, and R. Signorell, Phys. Chem. Chem. Phys. 20, 16364 (2018).

[30] S. Hartweg, B. L. Yoder, G. A. Garcia, L. Nahon, and R. Signorell, Phys. Rev. Lett. 118, 103402 (2017).

[31] R. Naaman, A. Haran, A. Nitzan, D. Evans, and M. Galperin, J. Phys. Chem. B 102, 3658 (1998). 
[32] K. Hiraoka and M. Nara, Bull. Chem. Soc. Jpn. 54, 1589 (1981).

[33] A. Nitzan, Annu. Rev. Phys. Chem. 52, 681 (2001).

[34] C. Herring and M. H. Nichols, Rev. Mod. Phys. 21, 185 (1949).

[35] P. Cutler and J. Davis, Surf. Sci. 1, 194 (1964).

[36] C. R. Crowell and S. M. Sze, J. Appl. Phys. 37, 2683 (1966).

[37] C. Bouchard and J. Carette, Surf. Sci. 100, 251 (1980).

[38] J.-P. Jay-Gerin and J. Karouni, Solid State Commun. 48, 69 (1983).

[39] C. B. Harris, N.-H. Ge, R. L. Lingle, J. D. McNeill, and C. M. Wong, Annu. Rev. Phys. Chem. 48, 711 (1997).

[40] T. Goulet, A. Bernas, C. Ferradini, and J.-P. Jay-Gerin, Chem. Phys. Lett. 170, 492 (1990).

[41] J. V. Coe, A. D. Earhart, M. H. Cohen, G. J. Hoffman, H. W. Sarkas, and K. H. Bowen, J. Chem. Phys. 107, 6023 (1997).

[42] W. Chen, F. Ambrosio, G. Miceli, and A. Pasquarello, Phys. Rev. Lett. 117, 186401 (2016).

[43] A. P. Gaiduk, T. A. Pham, M. Govoni, F. Paesani, and G. Galli, Nat. Commun. 9, 247 (2018).

[44] C. G. Elles, A. E. Jailaubekov, R. A. Crowell, and S. E. Bradforth, J. Chem. Phys. 125, 044515 (2006).

[45] A. Bernas, C. Ferradini, and J.-P. Jay-Gerin, Chem. Phys. 222, 151 (1997).

[46] R. M. Marsolais, M. Deschênes, and L. Sanche, Rev. Sci. Instrum. 60, 2724 (1989).

[47] M. Michaud, E. M. Hébert, P. Cloutier, and L. Sanche, J. Chem. Phys. 126, 024701 (2007).

[48] H. Burtscher, L. Scherrer, H. C. Siegmann, A. Schmidt-Ott, and B. Federer, J. Appl. Phys. 53, 3787 (1982).

[49] R. T. Nishida, A. M. Boies, and S. Hochgreb, J. Appl. Phys. 121, 023104 (2017).

[50] M. Grimm, B. Langer, S. Schlemmer, T. Lischke, U. Becker, W. Widdra, D. Gerlich, R. Flesch, and E. Rühl, Phys. Rev. Lett. 96, 066801 (2006).

[51] See Supplemental Material at http://link.aps.org/ supplemental/10.1103/PhysRevLett.124.013402 for experimental details, additional information on the scattering model, and additional figures, which includes Refs. [2,9,17,19,21,22,52-85].

[52] P. Liu, P. J. Ziemann, D. B. Kittelson, and P. H. McMurry, Aerosol Sci. Technol. 22, 293 (1995).

[53] P. Liu, P. J. Ziemann, D. B. Kittelson, and P. H. McMurry, Aerosol Sci. Technol. 22, 314 (1995).

[54] B. L. Yoder, A. H. C. West, B. Schläppi, E. Chasovskikh, and R. Signorell, J. Chem. Phys. 138, 044202 (2013).

[55] B. Dick, Phys. Chem. Chem. Phys. 16, 570 (2014).

[56] F. P. Schwarz and M. Mautner, Chem. Phys. Lett. 85, 239 (1982).

[57] T. W. Scott, C. L. Braun, and A. C. Albrecht, J. Chem. Phys. 76, 5195 (1982).
[58] V. O. Saik and S. Lipsky, J. Phys. Chem. 98, 11858 (1994).

[59] T. Goulet and J. P. Jay-Gerin, Solid State Commun. 55, 619 (1985).

[60] T. Goulet, V. Pou, and J.-P. Jay-Gerin, J. Electron Spectrosc. Relat. Phenom. 41, 157 (1986).

[61] R. Signorell, B. L. Yoder, A. H. C. West, J. J. Ferreiro, and C.-M. Saak, Chem. Sci. 5, 1283 (2014).

[62] A. Wiedensohler, J. Aerosol Sci. 19, 387 (1988).

[63] E. Knutson and K. Whitby, J. Aerosol Sci. 6, 443 (1975).

[64] M. Stolzenburg, An ultrafine aerosol size distribution measuring system, Ph.D. thesis, University of Minnesota, 1988.

[65] M. Alonso and Y. Kousaka, J. Aerosol Sci. 27, 1201 (1996).

[66] M. Alonso, F. Alguacil, and Y. Kousaka, J. Aerosol Sci. 31, 233 (2000).

[67] R. P. Camata, H. A. Atwater, and R. C. Flagan, J. Aerosol Sci. 32, 583 (2001).

[68] C. Zheng, Q. Chang, Q. Lu, Z. Yang, X. Gao, and K. Cen, Aerosol Air Qual. Res. 16, 3037 (2016).

[69] Y. Boisdron and J. Brock, Atmos. Environ. 4, 35 (1970).

[70] G. Biskos, E. Mastorakos, and N. Collings, J. Aerosol Sci. 35, 707 (2004).

[71] G. Biskos, K. Reavell, and N. Collings, J. Aerosol Sci. 36, 247 (2005).

[72] P. Arendt and H. Kallmann, Z. Phys. 35, 421 (1926).

[73] M. Pauthenier and M. Moreau-Hanot, J. Phys. Radium 3, 590 (1932).

[74] N. A. Fuchs, Izv. Akad. Nauk SSSR. Seriia Geogr. i Geofiz. 11, 341 (1947).

[75] P. J. Bricard, J. Geophys. Res. 54, 39 (1949).

[76] R. Gunn, J. Meteorol. 11, 339 (1954).

[77] T. Takahashi and Y. Harada, Bull. Chem. Soc. Jpn. 54, 81 (1981).

[78] M. Frisch et al., Gaussian 09, Revision D.01, (Gaussian Inc., Wallingford CT, 2013).

[79] L. Sanche, G. Bader, and L. Caron, J. Chem. Phys. 76, 4016 (1982).

[80] L. Sanche, J. Chem. Phys. 71, 4860 (1979).

[81] W. E. Wallace et al., Infrared Spectra in NIST Chemistry WebBook, NIST Standard Reference Database Number 69, (National Institute of Standards and Technology, Gaithersburg, MD, 2019).

[82] M. A. Yurkin and A. G. Hoekstra, J. Quant. Spectrosc. Radiat. Transfer 112, 2234 (2011).

[83] L. D. Landau and E. M. Lifshitz, Mechanics, 3rd ed. (Butterworth-Heinemann, London, 1976).

[84] A. Roy, R. Seidel, G. Kumar, and S. E. Bradforth, J. Phys. Chem. B 122, 3723 (2018).

[85] Y. Ando and T. Itoh, J. Appl. Phys. 61, 1497 (1987). 